\title{
Chronobiology and Sleep on Resilience
}

\author{
Hong Jun Jeon ${ }^{1}$ and So-Jin Lee ${ }^{2}$ \\ 'Department of Psychiatry, Konkuk University Medical Center, Seoul, Korea \\ ${ }^{2}$ Department of Psychiatry, Gyeongsang National University Hospital, Jinju, Korea
}

\begin{abstract}
Resilience refers to an ability to cope with adversities. Previous studies revealed that resilience works as a protective factor for various psychological conditions such as depression, anxiety, substance abuse and suicide. In the last decade, there has been numbers of studies focused on sleep and resilience in various populations. Insomnia has been reported to be a risk factor for depression, anxiety, post-traumatic stress disorder and suicide. In addition to insomnia, various sleep disturbance affects resilience in terms of sleep quality and sleep duration. Also, eveningness, exposure to chronic jetlag and circadian misalignment are risk factors for psychological problems and mental disorders. Further studies are needed to determine the effect of promoting healthy sleep behaviors on resilience.

Key Words: Resilience; Sleep; Mental health; Circadian rhythm; Jetlag

Received: November 5, 2019 Revised: December 7, 2019 Accepted: December 9, 2019

Corresponding author: So-Jin Lee, MD, PhD, Department of Psychiatry, Gyeongsang National University Hospital, 15 Jinju-daero, 816beon-gil, Jinju 52727, Korea.

Tel: 82-55-750-8921, Fax: 82-55-759-0003, E-mail: lifeofzoe@gnu.ac.kr

(a) This is an Open Access article distributed under the terms of the Creative Commons Attribution Non-Commercial License (https://creativecommons.org/licenses/bync/4.0) which permits unrestricted non-commercial use, distribution, and reproduction in any medium, provided the original work is properly cited.
\end{abstract}

\section{INTRODUCTION}

Resilience can be defined as one's ability to adapt to changes which is related to the inherent capacity to facing disturbances and make a new homeostasis [1]. Evidence shows that resilience work as a protective factor for numbers of psychiatric disorders including depression [2,3], anxiety [4], post-traumatic stress disorder (PTSD) [5], substance abuse [6], and suicide attempt [7]. As such, resilience became a valuable target in the field of psychiatry. Determinants of individual's resilience has received great attention from clinicians and researchers and several biological, environmental, genetic and psychological factors that affects human's resilience has been reported [8]. Among them, the relationship between resilience and sleep are getting more attention during the past decades.

Sleep is defined electroencephalographically, however, it encompasses variety of components such as duration, structure, phase and subjective quality. In some cases, it comes with pathological problems related with neurological or respiratory nature. With increasing interest in promoting resilience, how sleep increase or hinder one's resilience is a worthwhile subject to study. In this article, we will discuss the various aspects of sleep, such as sleep quality, circadian rhythm, circadian misalignment, and resilience.

\section{SLEEP QUALITY AND RESILIENCE}

A study on Swiss adolescents showed higher levels of sleep quality in those with greater mental toughness than those with weaker mental toughness [9]. High mental toughness is associated with better resilience and performance as well as sleep quality [10]. Also, Brand et al. [9] reported that subjects with greater mental toughness shows greater sleep efficiency, lesser awakening during sleep, shorter stage 1 sleep and longer slow wave sleep and rapid eye movement (REM) sleep. In the group with high mental toughness, subjective sleep quality as measured by the insomnia severity scale was also better, and the degree of daytime sleepiness as measured by the Epworth Sleepiness Scale was lower. Interestingly, there was no significant association between mental toughness and sleep latency nor sleep duration. The results of this study suggests that there is a bidirectional link between sleep and mental toughness. Until now, mechanism in such link has not been studied, however, the result of a large-scale prospective study that investigated the relation between insomnia symptoms and dysfunctional health behaviors partly support the bidirectional association between sleep and resilience [11].

Another study on the association between sleep disturbances and resilience in children and adolescents reported that sleep 
problems predict resilience and that resilience is a significant mediator in the process of manifesting internalization and externalization behavioral symptoms from insufficient sleep [12]. In this study, sleep disturbances were measured by the Sleep Disturbance Scale for Children, which includes questions on the sleep initiation, sleep maintenance, sleep disordered breathing, arousals during sleep, nightmares and excessive sleepiness during daytime. Resilience was measured through a self-report questionnaire called Resiliency Scales for Children and Adolescents. The result of this study suggests that sleep may improve neuroplasticity and eventually promote. Consistent with this, it was proposed that sleep decreased synaptic strength to a energetically sustainable level to make efficient use of gray matter space [13]. To conclude, it is possible that sufficient sleep enables individual to be resilient to adversity through maintaining neuroplasticity.

\section{SLEEP DURATION AND RESILIENCE}

In addition to sleep quality, sleep duration may affects one's resilience. Soldiers who already had symptoms of insomnia prior to deployment had a significantly higher risk of PTSD, depression, and anxiety after deployment. Short sleep duration less than 6 hours were also associated with the risk of PTSD symptoms [14]. However, in the studies on the relationship between mental toughness and sleep, sleep duration and sleep efficiency were not significantly related to mental toughness [9]. Besides it was reported that healthy female adolescents at risk for major depressive disorder did not show objectively measured shorter sleep duration, but only poorer quality of sleep than those without a risk for major depression [15]. Serum epinephrine level was correlated with sleep efficiency and interval between arousal in slow wave sleep, not with duration of slow wave sleep in partial slow wave sleep deprived healthy adults [16]. However, Irwin et al. [17] experimentally woke up healthy adults during $3 \mathrm{AM}$ to $6 \mathrm{AM}$ and found that serum epinephrine level was lower in slow wave sleep than in other sleep stages or awake. Another study by Ekstedt et al. [18], stressrelated physiological changes were related with sleep fragmentation rather than sleep duration or sleep efficiency. Hypothalamicpituitary-adrenal (HPA) axis is also responsible in the relation between resilience and sleep duration. Vgontzas et al. [19], reported that sleep deprivation decreased cortisol secretion the next day, which is caused by the increase of slow wave sleep during the recovery night. Chronic sleep deprivation also affects individual's reaction to stress [20]. Consistent with this, serotonin$1 \mathrm{~A}$ receptor was desensitized by sleep restriction [21].

\section{CHRONOTYPE AND RESILIENCE}

The association between circadian preference and resilience has been reported in a recent study [22]. This study was conducted on 1922 students and workers in Spain, used the reduced Morningness-Eveningness Questionnaire and the 10-item Connor-Davidson resilience scale [22]. Subjects with morning-type showed the highest score on the resilience scale in the morning followed by neither-type and evening-type. the lowest in the middle and evening [22]. Veterans with PTSD reports more symptoms of PTSD, more sleep disturbances and worse nightmares [23]. In addition, the activity of the brainstem which generates REM sleep and arousal is increased during sleep in evening-type subjects.

Since eveningness is associated with poor sleep quality [11,23,24], it is possible that the quality of sleep mediates the role chronotype in resilience. In addition, evening-type subjects are more likely to suffer from social jetlags that comes from desynchronization between biological clock and social clock $[22,25]$ and such jetlags may decreased individual's resilience. In a prospective study on college students in Hong Kong, evening-type students who live off campus showed lower quality of life than morning-type offcampus-living students [26]. Morning-type students who live on campus had lower quality of life than students who live off campus and were more likely to move to off-campus. It was suggested that either participation to night-life of campus or social withdrawal caused by not-participating to such activities may have decreased well-being of morning-type campus residents.

When experimental chronic jetlag was induced in female hamsters, asynchronous occurred between the internal clock and the time of the external environment, and the proliferation and neurogenesis of the hippocampus was suppressed [27]. However, the effect of jetlag on cell proliferation disappeared when adrenal grand was removed suggesting misalignment of time clocks is mediated by HPA axis. In terms of the cortisol level, experimentally induced jetlag affected as much as a significant stress did in the hamsters from same species [27,28]. After 8 days from the jetlag started, cortisol level was lowered than that of hamsters on stress, however, it was still higher than cortisol level before the jetlag [27,29]. These results are consistent with that female flight attendants who are exposed to frequent jetlag shows chronically increased level of cortisol and significantly lower volume of the right temporal lobe [30]. In this study, the degree of temporal lobe volume reduction and frequency of exposure to jetlag was associated with decrease in visuospatial cognitive function. The neurogenesis of these brain regions are known to have a role on affective regulation as well as cognitive function [31], therefore, it is possible that these regions are significantly related with resilience.

\section{CIRCADIAN MISALIGNMENT AND RESILIENCE}

It was suggested that circadian misalignment may have an impact on structure and function of prefrontal cortex. Karatsoreos et al. [32] exposed mice 20-hour light dark cycle and compared them with controls that were exposed normal 24-hour light dark cycle. The artificially induced circadian disruption yield volume reduction prelimbic medical prefrontal cortex in affected mice. This reduction was associated with decrease in cognitive flexibility and more impulsive behavior [32,33] which could be related to impairment of resilience. However, further studies are needed. 


\section{CONCLUSION}

Sleep characteristics affecting resilience are modifiable through adjustment of external environment or education on sleep hygiene, therefore it is worthy to examine the relationship between resilience and sleep. Highly resilient individuals do not easily progress to PTSD or major depressive disorder because they have the ability to withstand and overcome life adversities and crises and to recover or even achieve growth [34]. As discussed above, sleep-related factors such as sleep quality, sleep time, and circadian rhythm were associated with resilience. Although the mechanisms underlying the relationship between sleep and resilience have not been elucidated, there is a possibility of promoting resilience by improving sleep. Further research will be needed to see how improving the quality of sleep, having a good night's sleep, and maintaining a circadian rhythm on a personal schedule will have a positive impact on resilience.

\section{Acknowledgments}

This study was supported by a grant from the Korean Mental Health Technology R\&D Project, Ministry of Health \& Welfare, Republic of Korea (HM15C1108).

\section{Conflicts of Interest}

The authors have no potential conflicts of interest to disclose.

\section{Author Contributions}

Conceptualization: So-Jin Lee. Funding acquisition: So-Jin Lee. Investigation: So-Jin Lee, Hong Jun Jeon. Project administration: So-Jin Lee, Hong Jun Jeon. Resources: So-Jin Lee, Hong Jun Jeon. Supervision: So-Jin Lee. Writing_original draft: So-Jin Lee. Writing-review \& editing: So-Jin Lee, Hong Jun Jeon.

\section{ORCID iDs}

So-Jin Lee 1
https://orcid.org/0000-0003-2904-9206
Hong Jun Jeon (1)
https://orcid.org/0000-0002-0260-0494

\section{REFERENCES}

1. Germain A, Dretsch M. Sleep and resilience-a call for prevention and intervention. Sleep 2016;39:963-965.

2. Catalano D, Chan F, Wilson L, Chiu CY, Muller VR. The buffering effect of resilience on depression among individuals with spinal cord injury: a structural equation model. Rehabil Psychol 2011;56:200-211.

3. Sen S, Kranzler HR, Krystal JH, Speller H, Chan G, Gelernter J, et al. A prospective cohort study investigating factors associated with depression during medical internship. Arch Gen Psychiatry 2010;67:557-565.

4. Min JA, Lee NB, Lee CU, Lee C, Chae JH. Low trait anxiety, high resilience, and their interaction as possible predictors for treatment response in patients with depression. J Affect Disord 2012;137:61-69.

5. Lee JS, Ahn YS, Jeong KS, Chae JH, Choi KS. Resilience buffers the impact of traumatic events on the development of PTSD symptoms in firefighters. J Affect Disord 2014;162:128-133.

6. Fadardi JS, Azad H, Nemati A. The relationship between resilience, motivational structure, and substance use. Procedia Soc Behav Sci 2010;5:1956-1960.

7. Roy A, Sarchiapone M, Carli V. Low resilience in suicide attempters. Arch Suicide Res 2007;11:265-269.

8. Connor KM, Zhang W. Recent advances in the understanding and treatment of anxiety disorders. Resilience: determinants, measurement, and treatment responsiveness. CNS Spectr 2006;11(10 Suppl 12):5-12.

9. Brand S, Gerber M, Kalak N, Kirov R, Lemola S, Clough PJ, et al. Adolescents with greater mental toughness show higher sleep efficiency, more deep sleep and fewer awakenings after sleep onset. J Adolesc Health 2014;54: 109-113.

10. Gerber M, Brand S, Feldmeth AK, Lang C, Elliot C, Holsboer-Trachsler E, et al. Adolescents with high mental toughness adapt better to perceived stress: a longitudinal study with Swiss vocational students. Pers Individ Dif 2013; 54:808-814.

11. Merikanto I, Kronholm E, Peltonen M, Laatikainen T, Lahti T, Partonen T. Relation of chronotype to sleep complaints in the general Finnish population. Chronobiol Int 2012;29:311-317.

12. Chatburn A, Coussens S, Kohler MJ. Resiliency as a mediator of the impact of sleep on child and adolescent behavior. Nat Sci Sleep 2013;6:1-9.

13. Tononi G, Cirelli C. Sleep function and synaptic homeostasis. Sleep Med Rev 2006;10:49-62.

14. Gehrman P, Seelig AD, Jacobson IG, Boyko EJ, Hooper TI, Gackstetter GD, et al. Predeployment sleep duration and insomnia symptoms as risk factors for new-onset mental health disorders following military deployment. Sleep 2013;36:1009-1018.

15. Chen MC, Burley HW, Gotlib IH. Reduced sleep quality in healthy girls at risk for depression. J Sleep Res 2012;21:68-72.

16. Tiemeier H, Pelzer E, Jönck L, Möller HJ, Rao ML. Plasma catecholamines and selective slow wave sleep deprivation. Neuropsychobiology 2002;45:81-86.

17. Irwin M, Thompson J, Miller C, Gillin JC, Ziegler M. Effects of sleep and sleep deprivation on catecholamine and interleukin-2 levels in humans: clinical implications. J Clin Endocrinol Metab 1999;84:1979-1985.

18. Ekstedt M, Akerstedt T, Söderström M. Microarousals during sleep are associated with increased levels of lipids, cortisol, and blood pressure. Psychosom Med 2004;66:925-931.

19. Vgontzas AN, Mastorakos G, Bixler EO, Kales A, Gold PW, Chrousos GP. Sleep deprivation effects on the activity of the hypothalamic-pituitary-adrenal and growth axes: potential clinical implications. Clin Endocrinol (Oxf) 1999;51:205-215.

20. Meerlo P, Sgoifo A, Suchecki D. Restricted and disrupted sleep: effects on autonomic function, neuroendocrine stress systems and stress responsivity. Sleep Med Rev 2008;12:197-210.

21. Roman V, Hagewoud R, Luiten PG, Meerlo P. Differential effects of chronic partial sleep deprivation and stress on serotonin-1A and muscarinic acetylcholine receptor sensitivity. J Sleep Res 2006;15:386-394.

22. Antúnez JM, Navarro JF, Adan A. Circadian typology is related to resilience and optimism in healthy adults. Chronobiol Int 2015;32:524-530.

23. Hasler BP, Insana SP, James JA, Germain A. Evening-type military veterans report worse lifetime posttraumatic stress symptoms and greater brainstem activity across wakefulness and REM sleep. Biol Psychol 2013;94:255-262.

24. Yun JA, Ahn YS, Jeong KS, Joo EJ, Choi KS. The relationship between chronotype and sleep quality in Korean firefighters. Clin Psychopharmacol Neurosci 2015;13:201-208.

25. Wittmann M, Dinich J, Merrow M, Roenneberg T. Social jetlag: misalignment of biological and social time. Chronobiol Int 2006;23:497-509.

26. Lau EY, Wong ML, Ng EC, Hui CC, Cheung SF, Mok DS. "Social jetlag" in morning-type college students living on campus: implications for physical and psychological well-being. Chronobiol Int 2013;30:910-918.

27. Gibson EM, Wang C, Tjho S, Khattar N, Kriegsfeld LJ. Experimental 'jet lag' inhibits adult neurogenesis and produces long-term cognitive deficits in female hamsters. PLoS One 2010;5:e15267.

28. Jasnow AM, Drazen DL, Huhman KL, Nelson RJ, Demas GE. Acute and chronic social defeat suppresses humoral immunity of male Syrian hamsters (Mesocricetus auratus). Horm Behav 2001;40:428-433.

29. Meyer-Bernstein EL, Jetton AE, Matsumoto SI, Markuns JF, Lehman MN, Bittman EL. Effects of suprachiasmatic transplants on circadian rhythms of neuroendocrine function in golden hamsters. Endocrinology 1999;140:207-218.

30. Cho K. Chronic 'jet lag' produces temporal lobe atrophy and spatial cognitive deficits. Nat Neurosci 2001;4:567-568.

31. Samuels BA, Hen R. Neurogenesis and affective disorders. Eur J Neurosci 2011;33:1152-1159.

32. Karatsoreos IN, Bhagat S, Bloss EB, Morrison JH, McEwen BS. Disruption of circadian clocks has ramifications for metabolism, brain, and behavior. Proc Natl Acad Sci U S A 2011;108:1657-1662.

33. Karatsoreos IN. Effects of circadian disruption on mental and physical health. Curr Neurol Neurosci Rep 2012;12:218-225.

34. Jung YE, Chae JH. Neurobiology of resilience to cope against the life adversity. Korean J Psychophamacol 2010;21:62-70. 